\title{
Bayesian Inference of Equilibrium Magnetic Field Geometry on the MAST Experiment
}

\author{
Gregory T. von Nessi
}

\begin{abstract}
Inference of plasma equilibrium geometry in tokamak fusion plasmas constitutes a challenging inference problem, given intrinsic difficulties surrounding the making of direct measurements in such physical systems. Traditionally, this problem has been handled by codes that attempt to reconcile solutions of the Grad-Shafranov (GS) equation with external magnetic diagnostics. Due to this inference being an intrinsically ill-posed problem, these codes suffer from numerical difficulties that require experiment-specific algorithms to handle. Here, we present a method to directly infer plasma equilibrium structure based on Bayesian analysis, which does not require solving the GS equation nor the use of any experiment-specific numerics.
\end{abstract}

Index Terms-Fusion reactors, nuclear and plasma sciences, tokamaks, tokamak devices.

$\mathbf{K}$ NOWING the equilibrium magnetic field geometry of discharge plasmas is of central importance in the operation of modern tokamak fusion experiments. In particular, this knowledge is needed for actively controlling/shaping the plasma during a discharge and for the extraction of meaningful scientific data from many diagnostics present in modern fusion devices [2]. As modern tokamaks contain plasmas having temperatures on the order of $10^{7} \mathrm{~K}$, measuring this structure directly is generally not possible. Thus, the equilibrium field has traditionally been inferred by reconciling external magnetic diagnostic measurements with a force-balance constraint, manifested as an elliptic semilinear PDE called the Grad-Shafranov (GS) equation in axisymmetric systems (e.g., tokamaks) [2], [3]. Given that the external diagnostic measurements are finite in number, they are unable to give a complete boundary condition to uniquely determine a solution of the GS equation. The consequence of the ill-posed nature of this problem is that the numerical reconciliation of the GS equation with diagnostic measurements often needs experiment-specific algorithms to ensure that the numerical iterations converge to a physically relevant solution. Moreover, such numerical schemes have no intrinsic means by which to give uncertainties on the inferred equilibrium field.

Manuscript received November 29, 2010; revised July 14, 2011; accepted July 15, 2011. Date of publication August 30, 2011; date of current version November 9, 2011. This work was supported in part by the Australian Government through International Science Linkages under Grant CG130047, by the Australian National University, by the U.K. Engineering and Physical Sciences Research Council under Grant EP/G003955, and by the European Communities under the contract of association between EURATOM and CCFE.

The author is with the Research School of Physics and Engineering, Australian National University, Canberra, A.C.T. 0200, Australia.

Digital Object Identifier 10.1109/TPS.2011.2162967
Svensson and Werner developed a new way to infer equilibrium field structure, without force balance, using Bayesian analysis on the Joint European Tokamak [4]. This work has recently been expanded and used on the Mega Ampere Spherical Tokamak (MAST) [5], [6]. The analysis models the plasma as a collection of axisymmetric current beams, each of finite cross section, with a degree of smoothness imposed across the beam collection. Bayesian techniques are employed to set the strength of the smoothening constraint so as to not over- or underfit the data. The beam currents are inferred from external magnetic diagnostic and internal motional Stark effect (MSE) measurements via Bayesian analysis applied to Biot-Savart calculations and a linear MSE model. The error on the diagnostic measurements manifests as a probability distribution for each beam current in the final Bayesian inference. The magnetic field structure can subsequently be calculated from any sample taken from the beam current distributions by application of Ampere's law. Thus, sampling statistics are used to calculate uncertainties and the expectation of the equilibrium field. Even though uniqueness issues are circumvented, the uncertainties in a Bayesian inference will generally increase as the problem becomes more underdetermined.

A key advantage of this technique is that the inference is intrinsically analytic, in a sense that the inferred current distribution is multivariate Gaussian, with each dimension of the distribution corresponding to a particular beam current. To calculate and analyze statistics on this distribution take less than $1 \mathrm{~s}$ to complete on most modern desktop computers. Given the intrinsically low computational overhead of this analysis, realtime applications of this technique to plasma control are a focus of current research.

Fig. 1 shows the results of using the previously described Bayesian technique to infer the poloidal flux function structure on MAST discharge \#22254 at $350 \mathrm{~ms}$ : the time of peak $\beta$. Discharge \#22254 is a deuterium plasma in a double-null configuration heated with $3.1 \mathrm{MW}$ of neutral beam power. Fig. 1(a) shows a contour plot of the expectation of the poloidal flux function. Fig. 1(b) shows the uncertainties on the 0.25, 0.5, 0.75 , and 0.99 normalized flux surfaces generated by taking 200 samples from the plasma current distribution and plotting the density of the resulting flux contours. The raw data for the images were generated by a code written in MINERVA [7], a Java-based Bayesian framework. The data were plotted using standard routines in MATLAB.

In conclusion, images have been presented that demonstrate the success of using Bayesian analysis to infer the equilibrium magnetic field on a high-performance MAST discharge. This technique has advantages, in that it provides equilibrium field 


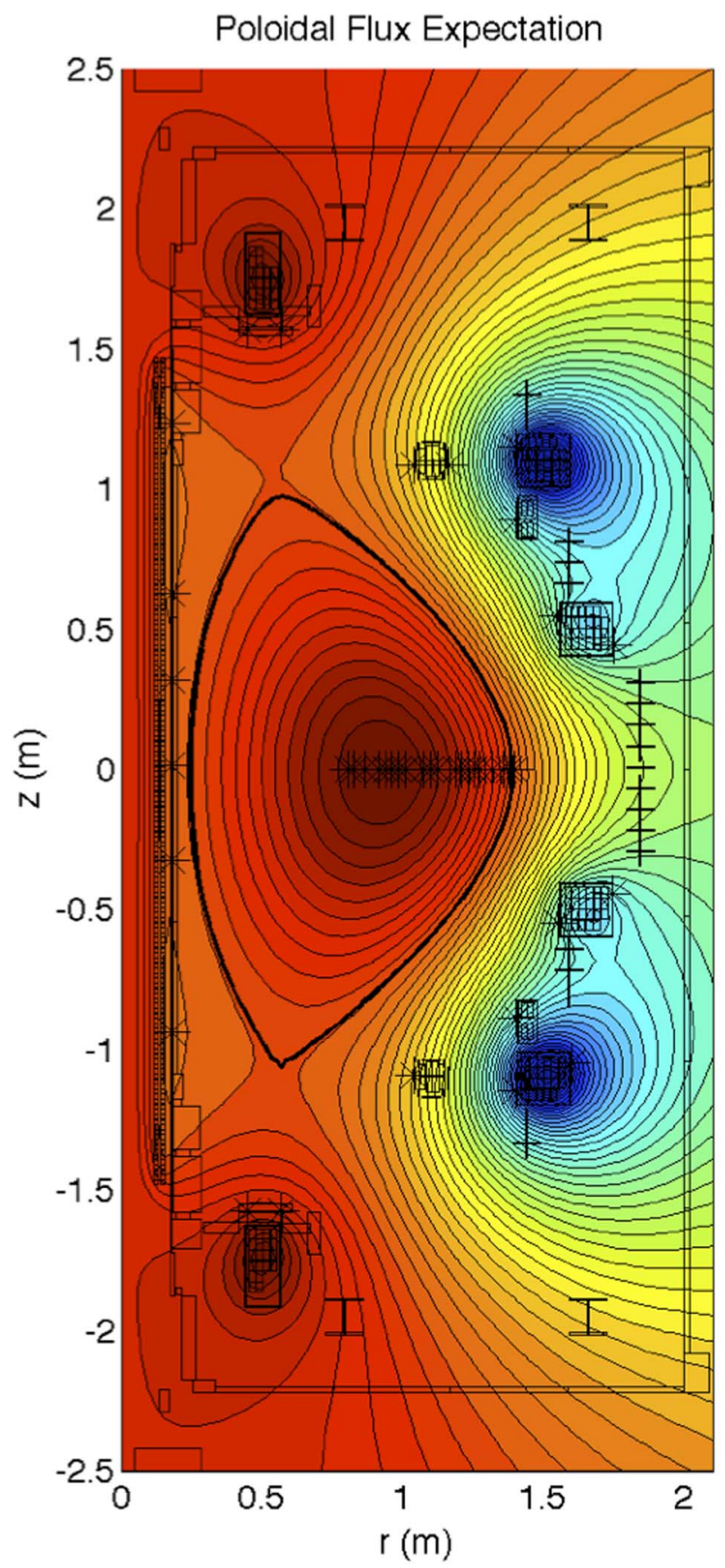

(a)

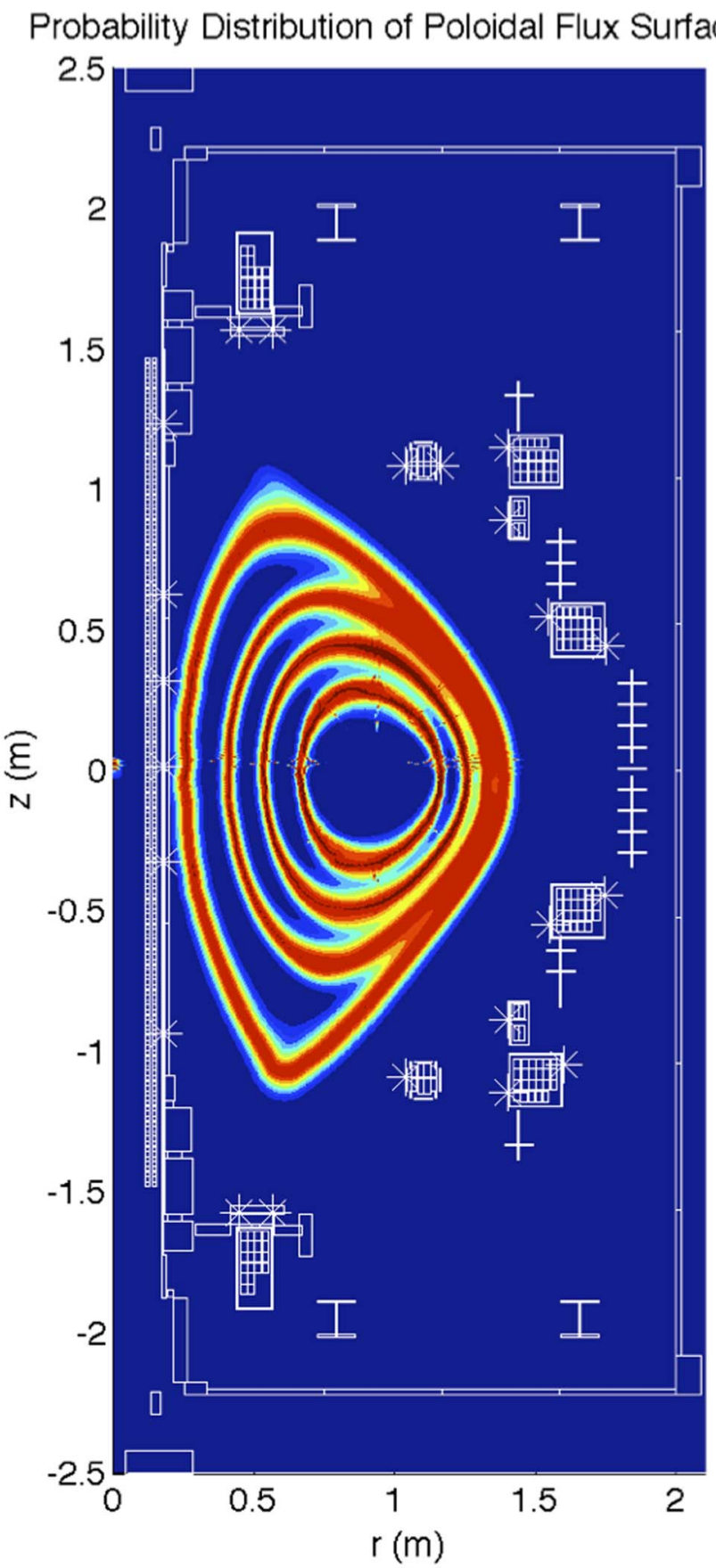

(b)

Fig. 1. Poloidal flux function contours for MAST discharge \#22254 at $350 \mathrm{~ms}$, mapped onto a poloidal cross section of the experiment. (a) Contour map of poloidal flux expectation. (b) Line density plot showing distributional uncertainties corresponding to the normalized poloidal flux contours of $0.25,0.50,0.75$, and 0.99 .

uncertainties and requires the use of only simple, fast, and robust numerical algorithms to work. Moreover, as the analysis does not use a force-balance constraint, it is complementary to GS solvers and is ideally suited for cross-validation.

\section{REFERENCES}

[1] L. Lao, H. St. John, R. Stambaugh, A. Kellman, and W. Pfeiffer, "Reconstruction of current profile parameters and plasma shapes in tokamaks," Nucl. Fusion, vol. 25, no. 11, pp. 1611-1622, Nov. 1985.

[2] J. Wesson, Tokamaks, 3rd ed. Oxford, U.K.: Oxford Univ. Press, 2004
[3] I. Hutchinson, Principles of Plasma Diagnostics, 2nd ed. Cambridge, U.K.: Cambridge Univ. Press, 2002.

[4] J. Svensson and A. Werner, "Current tomography for axisymmetric plasmas," Plasma Phys. Control. Fusion, vol. 50, no. 8, p. 085 002, Aug. 2008.

[5] M. Hole, G. von Nessi, J. Bertram, J. Svensson, L. Appel, B. Blackwell, R. Dewar, and J. Howard, "Model data fusion: Developing Bayesian inversion to constrain equilibrium and mode structure," J. Plasma Fusion Res., vol. 9, pp. 479-486, 2010 .

[6] M. Hole, G. von Nessi, D. Pretty, J. Howard, B. Blackwell, J. Svensson, and L. Appel, "The use of Bayesian inversion to resolve plasma equilibrium," Rev. Sci. Instrum., vol. 81, no. 10, p. 10E 127, Oct. 2010.

[7] J. Svensson and A. Werner, "Large scale Bayesian data analysis for nuclear fusion experiments," in Proc. IEEE Int. Symp. WISP, Oct. 2007, pp. 1-6. 